\title{
The quantum adversary method and classical formula size lower bounds
}

\author{
Sophie Laplante $^{*} \quad$ Troy Lee ${ }^{\dagger} \quad$ Mario Szegedy ${ }^{\ddagger}$
}

\begin{abstract}
We introduce two new complexity measures for Boolean functions, or more generally for functions of the form $f: S \rightarrow T$, where $S \subseteq \Sigma^{n}$ for some alphabet $\Sigma$ and $T$ an arbitrary set. We call these measures sumPI and maxPI. The quantity sumPI has been emerging through a line of research on quantum query complexity lower bounds via the so-called quantum adversary method Amb02 Amb03, BSS03 Zha04, LM04, culminating in SS04 with the realization that these many different formulations are in fact equivalent. Given that sumPI turns out to be such a robust invariant of a function, we begin to investigate this quantity in its own right and see that it also has applications to classical complexity theory.

As a surprising application we show that $\operatorname{sumPl}^{2}(f)$ is a lower bound on the formula size, and even, up to a constant multiplicative factor, the probabilistic formula size of $f$. We show that several formula size lower bounds in the literature, specifically Khrapchenko and its extensions Khr71, Kou93, including a key lemma of Hås98, are in fact special cases of our method.

The second quantity we introduce, $\max \operatorname{PI}(f)$, is always at least as large as sumPI $(f)$, and is derived from sumPI in such a way that $\operatorname{maxPI}^{2}(f)$ remains a lower bound on formula size. While sumPI $(f)$ is always a lower bound on the quantum query complexity of $f$, this is not the case in general for $\operatorname{maxPI}(f)$.

A strong advantage of sumPI $(f)$ is that it has both primal and dual characterizations, and thus it is relatively easy to give both upper and lower bounds on the sumPI complexity of functions. To demonstrate this, we look at a few concrete examples. We characterize the sumPI complexity of the height $h$ recursive majority of three function, which immediately gives a bound of $\Omega\left(2^{h}\right)$ for the bounded error quantum query complexity, and $4^{h}$ for the probabilistic formula size. We also characterize the sumPI complexity of a function defined by Ambainis Amb03 and show that for this function our method gives a much stronger lower bound on the formula size than is possible with Khrapchenko's method.

Finally, we characterize the sumPI and maxPI complexity of the collision problem and show this is an example where maxPI can be much larger than sumPI.
\end{abstract}

\section{Introduction}

A central and longstanding open problem in complexity theory is to prove superlinear lower bounds for the circuit size of an explicit Boolean function. While this seems quite difficult, a modest amount of success has been achieved in the slightly weaker model of formula size, a formula being simply

\footnotetext{
${ }^{*}$ LRI, Université Paris-sud laplante@lri.fr

${ }^{\dagger}$ CWI and University of Amsterdam Troy.Lee@cwi.nl

${ }^{\ddagger}$ Rutgers University szegedy@cs.rutgers . edu
} 
a circuit where every gate has fan-out at most one. The current best formula size lower bound for an explicit function is $n^{3-o(1)}$ by Håstad Hås98.

In this paper we show that part of the rich theory developed around proving lower bounds on quantum query complexity, namely the so-called quantum adversary argument, can be brought to bear on formula size lower bounds. This adds to the growing list of examples of how studying quantum computing has led to new results in classical complexity, including [SV01, KW03, Aar04, LM04, to cite just a few.

The roots of the quantum adversary argument can be traced to the hybrid argument of BBBV97, who use it to show a $\Omega(\sqrt{n})$ lower bound on quantum search. Ambainis went on to formulate his original unweighted and later weighted schemes Amb02, Amb03. Other generalizations include Barnum, Saks, and Szegedy BSS03. with their spectral method and Zhang Zha04] with his strong adversary method. Laplante and Magniez LM04 use Kolmogorov complexity to capture the adversary argument in terms of a minimization problem. This line of research culminates in recent work of Špalek and Szegedy ŠS04 who show that in fact all the methods of Amb03, BSS03, Zha04, LM04, are equivalent.

The fact that the quantum adversary argument has so many equivalent definitions indicates that it is a natural combinatorial property of Boolean functions which is worthwhile to investigate on its own. We give this quantity its own name, sumPI, and adopt the following primal formulation of the method, from SS04, LM04. Letting $f: S \rightarrow T$, where $S \subseteq \Sigma^{n}$ for some alphabet $\Sigma$, and $T$ is an arbitrary set, we say

$$
\operatorname{sumPI}(f)=\min _{p} \max _{\substack{x, y \\ f(x) \neq f(y)}} \frac{1}{\sum_{\substack{i \\ x_{i} \neq y_{i}}} \sqrt{p_{x}(i) p_{y}(i)}}
$$

where $p=\left\{p_{x}: x \in S\right\}$ is a family of probability distributions on the indices $[n]$. If $Q_{\epsilon}(f)$ is the $\epsilon$-error quantum query complexity of $f$ then $Q_{\epsilon}(f) \geq(1-2 \sqrt{\epsilon(1-\epsilon)})$ sumPI $(f)$. We show further that sumPI ${ }^{2}(f)$ is a lower bound on the formula size of $f$.

We also introduce $\mathrm{KI}(f)=\min _{\alpha \in \Sigma^{*}} \max _{\substack{x, y \\ f(x) \neq f(y)}} \min _{i: x_{i} \neq y_{i}} K(i \mid x, \alpha)+K(i \mid y, \alpha)$, where $K$ is prefix-free Kolmogorov complexity. This formulation arises from the quantum and randomized lower bounds of LM04. This formulation is especially interesting because of the intuition that it provides. For example, it allows for a very simple proof that circuit depth $\mathrm{d}(f) \geq \mathrm{KI}(f)$, using the Karchmer-Wigderson characterization of circuit depth KW88.

This does not immediately imply that $2^{\mathrm{KI}}$ is a lower bound on formula size, but it led us to speculate that this was true. We define a quantity closely related to $2^{\mathrm{KI}}$, which we call maxPI.

$$
\operatorname{maxPI}(f)=\min _{p} \max _{\substack{x, y \\ f(x) \neq f(y)}} \frac{1}{\max _{i: x_{i} \neq y_{i}} \sqrt{p_{x}(i) p_{y}(i)}} .
$$

Notice that this is like sumPI but where the sum is replaced by a maximum. By definition, maxPI is larger than sumPI, but its square is still a lower bound on formula size. However, maxPI is no longer a lower bound on quantum query complexity in general, and we give an example of a partial function $f$ for which sumPI $(f)=2$ and $\operatorname{maxPI}(f)=\sqrt{n / 2}$. For this function, the collision problem, $\max \mathrm{PI}(f) \gg Q_{\epsilon}(f)=\Theta\left(n^{1 / 3}\right)$ [AS04, BHT97.

Although maxPI $(f)$ is a larger quantity, there are several advantages to working with the quantity sumPI $(f)$. To lower bound sumPI $(f)$ it is often convenient to work with its formulation as 
a maximization problem obtained by dualizing Equation (11). Among the several expressions the most convenient for our purposes will be the following from [LM04]:

$$
\operatorname{sumPI}(f)=\max _{P} \min _{\substack{x, y, i \\ f(x) \neq f(y), x_{i} \neq y_{i}}} \frac{\sqrt{p_{A}(x) p_{B}(y) p_{x, i}^{\prime}(y) p_{y, i}^{\prime}(x)}}{q(x, y)},
$$

where $q$ is a probability distribution on $S \times S$ with the property that $q(x, y)=0$ when $f(x)=f(y)$. $p_{A}, p_{B}$ are probability distributions on $S$, and $\left\{p_{x, i}^{\prime}: x \in S, i \in[n]\right\}$ is a family of probability distributions on $S$, and $P$ ranges over all possible settings to the tuple $\left(q, p_{A}, p_{B},\left\{p_{x, i}^{\prime}\right\}_{x, i}\right)$ of distributions as above.

By using the different formulations Eq. 1 and Eq. 3 of sumPI appropriately, it is relatively easy to show that sumPI strongly generalizes several lower bound techniques in formula size, including the lower bound of Khrapchenko Khr71, Kou93, and even a key lemma of Håstad from the aforementioned $n^{3-o(1)}$ formula size lower bound result. A further advantage of sumPI is that the probabilistic formula size of $f$ is $\Omega\left(\operatorname{sumPI}^{2}(f)\right)$, which we do not know to be true for $\operatorname{max\mathrm {PI}^{2}}(f)$. As sumPI $(f)$ is a lower bound on quantum query complexity, and a related quantity is a lower bound on randomized query complexity, examining the quantity sumPI $(f)$ can give lower bounds in three computational models with a single analysis. On the other hand, this advantage also has a flip side that sumPI ${ }^{2}(f)$ is always less than $n^{2}$. The quantity $\operatorname{maxPI^{2}}(f)$ shares this same limitation.

We look at several concrete problems to illustrate the strengths and weaknesses of our methods. We study the height $h$ recursive majority of three problem, $\mathrm{R}-\mathrm{MAJ}_{3}^{h}$, and show that $Q_{\epsilon}\left(\mathrm{R}-\mathrm{MAJ}_{3}^{h}\right)=\Omega\left(2^{h}\right)$ and a lower bound of $4^{h}$ for the formula size. We also look at a function defined by Ambainis Amb03 to separate the quantum query complexity of a function from the bound given by the polynomial method $\left[\mathrm{BBC}^{+} 01\right.$. This function gives an example where sumPI ${ }^{2}$ can give something much better than Khraphchenko's bound. We also give bounds for the collision problem.

\section{$1.1 \quad$ Organization}

In Section 2, we give the definitions, results, and notation that we use throughout the paper, and introduce the quantities sumPI, maxPI, and KI. In Section 3. we show how sumPI and maxPI give rise to formula size lower bounds, for deterministic and probabilistic formula size. In Section 4 we prove some properties of sumPI and maxPI. In Section 5, we compare our new methods with previous methods in formula size complexity. In Section 6, we investigate the limits of our and other formula lower bound methods. Finally, in Section $[7$ we apply our techniques to some concrete problems.

\section{Preliminaries}

We use standard notation such as $[n]=\{1, \ldots, n\},|S|$ for the cardinality of a set $S$, and all logarithms are base 2 . Hamming distance is written $d_{H}$.

We will also use standard measures of Boolean functions, such as sensitivity and certificate complexity. We briefly recall these here, see BW02] for more details. For a set $S \subseteq\{0,1\}^{n}$ and Boolean function $f: S \rightarrow\{0,1\}$, the sensitivity of $f$ on input $x$ is the number of positions $i \in[n]$ such that changing the value of $x$ in position $i$ changes the function value. The zero-sensitivity, 
written $s^{0}(f)$ is the maximum over $x \in f^{-1}(0)$ of the sensitivity of $f$ on $x$. The one-sensitivity, $s^{1}(f)$ is defined analogously. The maximum of $s^{0}(f), s^{1}(f)$ is the sensitivity of $f$, written $s(f)$.

A certificate for $f$ on input $x \in S$ is a subset $I \subseteq[n]$ such that for any $y$ satisfying $y_{i}=x_{i}$ for all $i \in I$ it must be the case that $f(y)=f(x)$. The zero-certificate complexity of $f$, written $C_{0}(f)$ is the maximum over all $x \in f^{-1}(0)$ of the minimum size certificate of $x$. Similarly, the one-certificate complexity of $f$, written $C_{1}(f)$ is the maximum over all $x \in f^{-1}(1)$ of the minimum size certificate of $x$.

We will further need some terminology from linear algebra. For a matrix $A$ we write $A^{T}$ for the transpose of $A$, and $\lambda(A)$ for the spectral norm of $A$, that is the absolute value of the largest eigenvalue of $A$. For two matrices $A, B$ we let $A \circ B$ be the Hadamard product of $A$ and $B$, that is $(A \circ B)[x, y]=A[x, y] B[x, y]$. We write $A \geq B$ if $A$ is entrywise greater than $B$, and $A \succeq B$ when $A-B$ is positive semidefinite, that is $\forall v: v^{T}(A-B) v \geq 0$.

\subsection{Deterministic and probabilistic formulae}

A Boolean formula over the standard basis $\{\vee, \wedge, \neg\}$ is a binary tree where each internal node is labeled with $\vee$ or $\wedge$, and each leaf is labeled with a literal, that is, a Boolean variable or its negation. The size of a formula is its number of leaves. We naturally identify a formula with the function it computes.

Definition 1 Let $f:\{0,1\}^{n} \rightarrow\{0,1\}$ be a Boolean function. The formula size of $f$, denoted $\mathrm{L}(f)$, is the size of the smallest formula which computes $f$. The formula depth of $f$, denoted $\mathrm{d}(f)$ is the minimum depth of a formula computing $f$.

It is clear that $\mathrm{L}(f) \leq 2^{\mathrm{d}(f)}$; that in fact the opposite inequality $\mathrm{d}(f) \leq O(\log \mathrm{L}(f))$ also holds is a nontrivial result due to Spira Spi71.

We will also consider probabilistic formulae, that is, a probability distribution over deterministic formulae. We take a worst-case notion of the size of a probabilistic formula. Probabilistic formula size has been studied before, for example in Val84, Bop89, DZ97, Kla04.

Definition 2 Let $\left\{f_{j}\right\}_{j \in J}$ be a set of functions with $f_{j}: S \rightarrow T$ for each $j \in J$. For a function $f: S \rightarrow T$, we say that $f$ is $\epsilon$-approximated by $\left\{f_{j}\right\}_{j \in J}$ if there is a probability distribution $\alpha=$ $\left\{\alpha_{j}\right\}_{j \in J}$ over $J$ such that for every $x \in S$,

$$
\underset{\alpha}{\operatorname{Pr}}\left[f(x)=f_{j}(x)\right] \geq 1-\epsilon .
$$

In particular, if $\max _{j} \mathrm{~L}\left(f_{j}\right) \leq s$, then we say that $f$ is $\epsilon$-approximated by formulas of size $s$, denoted $\mathrm{L}^{\epsilon}(f) \leq s$.

Note that even if a function depends on all its variables, it is possible that the probabilistic formula size is less than the number of variables.

\subsection{Quantum Adversary Method}

A deep knowledge of quantum computing is not needed for reading this paper; for completeness, however, we briefly sketch the quantum query model. More background on quantum query complexity and quantum computing in general can be found in [BW02, NC00]. 
As with the classical counterpart, in the quantum query model we wish to compute some function $f: S \rightarrow\{0,1\}$, where $S \subseteq \Sigma^{n}$, and we access the bits of the input through queries. The complexity of $f$ is the number of queries needed to compute $f$. Unlike the classical case, however, we can now make queries in superposition. Formally, a query $O$ corresponds to the unitary transformation

$$
O:|i, b, z\rangle \mapsto\left|i, b \oplus x_{i}, z\right\rangle
$$

where $i \in[n], b \in\{0,1\}$, and $z$ represents the workspace. A $t$-query quantum algorithm $A$ has the form $A=U_{t} O U_{t-1} O \cdots O U_{1} O U_{0}$, where the $U_{k}$ are fixed unitary transformations independent of the input $x$. The computation begins in the state $|0\rangle$, and the result of the computation $A$ is the observation of the rightmost bit of $A|0\rangle$. We say that $A \epsilon$-approximates $f$ if the observation of the rightmost bit of $A|O\rangle$ is equal to $f(x)$ with probability at least $1-\epsilon$, for every $x$. We denote by $Q_{\epsilon}(f)$ the minimum query complexity of a quantum query algorithm which $\epsilon$-approximates $f$.

Along with the polynomial method $\mathrm{BBC}^{+} 01$, one of the main techniques for showing lower bounds in quantum query complexity is the quantum adversary method Amb02, Amb03, BSS03, Zha04, LM04. Recently, Špalek and Szegedy ŠS04 have shown that all the strong versions of the quantum adversary method are equivalent, and further that these methods can be nicely characterized as primal and dual. For our purposes, we will use one formulation of the primal method, and two formulations of the dual method. We take the following primal method, implicit in [LM04, and explicitly formulated and called the minimax method in SS04.

Definition 3 (Minimax) Let $n \geq 1$ be an integer, $S \subseteq \Sigma^{n}$ and $\left\{p_{x}: x \in S\right\}$ be a family of probability distributions over $[n]$. Then

$$
\operatorname{MM}(f)=\min _{p} \max _{\substack{x, y \\ f(x) \neq f(y)}} \frac{1}{\sum_{\substack{i \\ x_{i} \neq y_{i}}} \sqrt{p_{x}(i) p_{y}(i)}}
$$

The primal method is easy to upper bound as we simply have to exhibit a family of probability distributions. Thus for example we use the primal method to show that sumPI ${ }^{2}$ is upper bounded by formula size.

We will use two versions of the dual method, both a weight scheme and the spectral formulation. The most convenient weight scheme for us is the "probability scheme", given in Lemma 4 of [LM04].

Definition 4 (Probability Scheme) Let $\Sigma$ be a finite set, $n \geq 1$ an integer, and $S \subseteq \Sigma^{n}$. Let $q$ be a probability distribution on $S \times S$, let $p_{A}, p_{B}$ be probability distributions on $S$, and let $\left\{p_{x, i}^{\prime}: x \in S, i \in[n]\right\}$ be a family of probability distributions on $S$. Assume that $q(x, y)=0$ when $f(x)=f(y)$. Let $P$ range over all possible tuples $\left(q, p_{A}, p_{B},\left\{p_{x, i}^{\prime}\right\}_{x, i}\right)$ of distributions as above. Then

$$
\operatorname{PA}(f)=\max _{P} \min _{\substack{x, y, i \\ f\left(x \neq f(y), x_{i} \neq y_{i}\right.}} \frac{\sqrt{p_{A}(x) p_{B}(y) p_{x, i}^{\prime}(y) p_{y, i}^{\prime}(x)}}{q(x, y)}
$$

We will also use the spectral adversary method.

Definition 5 (Spectral Adversary) Let $\Gamma$ denote an arbitrary $|S| \times|S|$ non-negative symmetric matrix that satisfies $\Gamma[x, y]=0$ whenever $f(x)=f(y)$. For $i \in[n]$, let $\Gamma_{i}$ be the matrix:

$$
\Gamma_{i}[x, y]= \begin{cases}0 & \text { if } x_{i}=y_{i} \\ \Gamma[x, y] & \text { if } x_{i} \neq y_{i}\end{cases}
$$


Then

$$
\mathrm{SA}(f)=\max _{\Gamma} \frac{\lambda(\Gamma)}{\max _{i} \lambda\left(\Gamma_{i}\right)}
$$

We will often use the following theorem implicitly in taking the method most convenient for the particular bound we wish to demonstrate.

Theorem 6 (Špalek-Szegedy) Let $n \geq 1$ be an integer, $S \subseteq\{0,1\}^{n}$ and $f: S \rightarrow\{0,1\}$. Then

$$
\mathrm{MM}(f)=\mathrm{SA}(f)=\mathrm{PA}(f)
$$

\subsection{The sumPI and maxPI complexity measures}

Theorem [6 tells us that the measure MM is a robust invariant of functions. We highlight this by giving it a new name, sumPI. We also define a similar quantity, maxPI.

Definition 7 Let $f: S \rightarrow T$ be a function with $S \subseteq \Sigma^{n}$ and $T$ an arbitrary set. For every $x \in S$ let $p_{x}:[n] \rightarrow \mathbb{R}$ be a probability distribution, that $i s, p_{x}(i) \geq 0$ and $\sum_{i} p_{x}(i)=1$. Let $p=\left\{p_{x}: x \in S\right\}$. We define the sum probability of indices to be

$$
\operatorname{sumPI}(f)=\min _{p} \max _{\substack{x, y \\ f(x) \neq f(y)}} \frac{1}{\sum_{\substack{i \\ x_{i} \neq y_{i}}} \sqrt{p_{x}(i) p_{y}(i)}}
$$

We define the maximimum probability of indices to be

$$
\operatorname{maxPI}(f)=\min _{p} \max _{\substack{x, y \\ f(x) \neq f(y)}} \frac{1}{\max _{i} \sqrt{p_{x}(i) p_{y}(i)}}
$$

It can be easily seen from the definitions that $\operatorname{sumPI}(f) \leq \max \mathrm{PI}(f)$ for any $f$. The following lemma is also straightforward from the definitions:

Lemma 8 If $S^{\prime} \subseteq S$ and $f^{\prime}: S^{\prime} \rightarrow T$ is a domain restriction of $f: S \rightarrow T$ to $S^{\prime}$, then $\operatorname{sumPI}\left(f^{\prime}\right) \leq$ $\operatorname{sumPI}(f)$ and $\max \mathrm{PI}\left(f^{\prime}\right) \leq \max \mathrm{PI}(f)$.

\subsection{The KI complexity measure}

The definition of KI arises from the Kolmogorov complexity adversary method [LM04]. The Kolmogorov complexity $C_{U}(x)$ of a string $x$, with respect to a universal Turing machine $U$ is the length of the shortest program $p$ such that $U(p)=x$. The complexity of $x$ given $y$, denoted $C(x \mid y)$ is the length of the shortest program $p$ such that $U(\langle p, y\rangle)=x$. When $U$ is such that the set of outputs is prefix-free (no string in the set is prefix of another in the set), we write $K_{U}(x \mid y)$. From this point onwards, we fix $U$ and simply write $K(x \mid y)$. For more background on Kolmogorov complexity consult [LV97.

Definition 9 Let $S \subseteq \Sigma^{n}$ for an alphabet $\Sigma$, and $T$ be an arbitrary set. For any function $f: S \rightarrow T$, let

$$
\mathrm{KI}(f)=\min _{\alpha \in \Sigma^{*}} \max _{\substack{x, y \\ f(x) \neq f(y)}} \min _{i: x_{i} \neq y_{i}} K(i \mid x, \alpha)+K(i \mid y, \alpha)
$$

An argument similar to that in $\underline{\text { ŠS04 }}$ shows that $2^{\mathrm{KI}(f)}=\Theta\left(\max \mathrm{PI}^{2}(f)\right)$. The advantage of $\mathrm{KI}$ is that it naturally captures the information theoretic content of the adversary methods, and in many cases gives a more natural intuition than sumPI and maxPI. As an example of this, we will give a very simple proof that $\mathrm{KI}$ is a lower bound on circuit depth in Section 3.1 


\section{Formula Size Lower Bounds}

One of the most important open problems in complexity theory is proving a superlinear lower bound on circuit size, for an explicit function. By counting, functions must exist that require exponential size, and most have size at least $2^{n} / n$ Sha49, but to date the best lower bound known is $5 n-o(n)$ LR01, IM02. Better bounds are known for formula size, where the largest lower bound for an explicit function is $n^{3-o(1)}$ [Hås98].

\subsection{Lower bound on depth using $\mathrm{KI}$}

To illustrate the KI method, we prove a lower bound on circuit depth which highlights the relationship between the adversary methods and communication complexity.

For any relation $R(x, y, z)$, the communication complexity $\mathrm{D}(R)$ is the communication cost of the communication game where $A$ is given $x, B$ is given $y$, and their goal is to compute some $z$ such that $R(x, y, z)$ (if such a $z$ exists). Our proof uses the characterization of circuit depth given by Karchmer and Wigderson in terms of the communication complexity of the associated relation $R_{f}$.

Definition 10 For any Boolean function $f, R_{f}=\left\{(x, y, i): f(x) \neq f(y), x_{i} \neq y_{i}\right\}$.

Theorem 11 (Karchmer-Wigderson) For any Boolean function $f, \mathrm{~d}(f)=\mathrm{D}\left(R_{f}\right)$.

Theorem 12 Let $f$ be a Boolean function. Then $\mathrm{KI}(f) \leq \mathrm{d}(f)$.

Proof: Let $P$ be a protocol for $R_{f}$. Fix $x, y$ with different values under $f$, and let $T_{A}$ be a transcript of the messages sent from $\mathrm{A}$ to $\mathrm{B}$, and $T_{B}$ be a transcript of the messages sent from $\mathrm{B}$ to $\mathrm{A}$. Let $i$ be the output of the protocol, with $x_{i} \neq y_{i}$. To print $i$ given $x$, simulate $P$ using $x$ and $T_{B}$. To print $i$ given $y$, simulate $P$ using $y$ and $T_{A}$. This shows that $\forall x, y: f(x) \neq f(y), \exists i: x_{i} \neq$ $y_{i}, K(i \mid x, \alpha)+K(i \mid y, \alpha) \leq\left|T_{A}\right|+\left|T_{B}\right| \leq \mathrm{D}\left(R_{f}\right)$, where $\alpha$ is a description of $A$ 's and $B$ 's algorithms.

Remark A similar proof in fact shows that $\mathrm{KI}(f) \leq 2 \mathrm{~N}\left(R_{f}\right)$, where $N$ is the nondeterministic communication complexity. Since the bound does not take advantage of interaction between the two players, in many cases we cannot hope to get optimal lower bounds using these techniques.

\subsection{Deterministic Formulae}

We will use the following simple lemma which can be found in Wegener Weg87 where it is attributed to Paterson.

Lemma 13 Let $B_{n}$ be the set of functions $f:\{0,1\}^{n} \rightarrow\{0,1\}$. Suppose that $\mathrm{FC}: B_{n} \rightarrow \mathbb{N}$ satisfies the following three properties:

1. $\mathrm{FC}\left(x_{i}\right)=1$ for $1 \leq i \leq n$,

2. $\mathrm{FC}(f)=\mathrm{FC}(\neg f)$ for $f \in B_{n}$,

3. $\mathrm{FC}(f \vee g) \leq \mathrm{FC}(f)+\mathrm{FC}(g)$ for $f, g \in B_{n}$ 
Then $\mathrm{L}(f) \geq \mathrm{FC}(f)$ for any $f \in B_{n}$. We will call any function $\mathrm{FC}$ satisfying these properties a formal complexity measure.

These properties abstract a sufficient condition to show that FC is a lower bound on formula size by induction on the depth of the formula.

We now show that max $\mathrm{PI}^{2}$ is a formal complexity measure and thus both maxPl${ }^{2}$ and sumPI ${ }^{2}$ give lower bounds on formula size.

Theorem $14 \mathrm{~L}(f) \geq \operatorname{maxPl}^{2}(f) \geq \operatorname{sumPl}^{2}(f)$ for any Boolean function $f$.

Proof: We show that maxPI ${ }^{2}$ satisfies the three properties of Lemma 13

Item (1): Let $p$ be the distribution which for every $x$ puts all probability on $i$, that is, $p_{x}(i)=1$ for all $x$. Any $x, y$ with $f(x) \neq f(y)$ differ on position $i$, thus $\max \mathrm{PI}^{2}(f) \leq 1$. It is always the case that $\operatorname{maxPI^{2}}(f) \geq 1$ thus $\operatorname{maxPI^{2}}(f)=1$.

Item (2): This follows as maxPI${ }^{2}(f)$ is symmetric in $x$ and $y$.

Item (3): Let $\operatorname{maxPI}^{2}(f)=k$ and $\operatorname{maxPI}^{2}(g)=\ell$ and let $\{q\}_{x},\left\{q^{\prime}\right\}_{x}$ be sets of probability distributions realizing these bounds, respectively. That is,

- For every $x, y$ such that $f(x)=1$ and $f(y)=0, \min _{i} 1 /\left(q_{x}(i) q_{y}(i)\right) \leq k$

- For every $x, y$ such that $g(x)=1$ and $g(y)=0, \min _{i} 1 /\left(q_{x}^{\prime}(i) q_{y}^{\prime}(i)\right) \leq \ell$

We define a new set of probability distributions $\{p\}_{x}$ as follows:

- if $f(x)=1$, we set $p_{x}(i)=q_{x}(i)$ for all $i$

- if $f(x)=0$ and $g(x)=1$, then we set $p_{x}(i)=q_{x}^{\prime}(i)$ for all $i$

- if $f(x)=g(x)=0$ then we set $p_{x}(i)=\frac{k}{k+\ell} q_{x}(i)+\frac{\ell}{k+\ell} q_{x}^{\prime}(i)$ for all $i$.

Take any $x, y$ such that $f(x) \vee g(x)=1$ and $f(y) \vee g(y)=0$. It must be the case that at least one of $f(x)=1, g(x)=1$ holds. Suppose that $f(x)=1$. Then

$$
\begin{aligned}
\min _{i} \frac{1}{p_{x}(i) p_{y}(i)} & =\min _{i} \frac{1}{q_{x}(i)\left(\frac{k}{k+\ell} q_{y}(i)+\frac{\ell}{k+\ell} q_{y}(i)\right)} \\
& \leq \min _{i} \frac{k+\ell}{k q_{x}(i) q_{y}(i)} \leq k+\ell
\end{aligned}
$$

The case where $g(x)=1$ and $f(x)=0$ follows similarly.

\subsection{Probabilistic Formulae}

The nice properties of sumPI allow us to show further that it can be used to lower bound the probabilistic formula size.

Lemma 15 Let $\epsilon<1 / 2$. If $f: S \rightarrow T$ is $\epsilon$-approximated by functions $\left\{f_{j}\right\}_{j \in J}$ with $\operatorname{sumPI}\left(f_{j}\right) \leq s$ for every $j \in J$, then $\operatorname{sumPI}(f) \leq s /(1-2 \epsilon)$. 
Proof: By assumption there is a probability distribution $\alpha=\left\{\alpha_{j}\right\}_{j \in J}$ such that $\operatorname{Pr}\left[f(x)=f_{j}(x)\right] \geq$ $1-\epsilon$. Thus for a fixed $x \in S$, letting $J_{x}=\left\{j \in J: f(x)=f_{j}(x)\right\}$, we have $\sum_{j \in J_{x}} \alpha_{j} \geq 1-\epsilon$. Hence for any $x, y \in S$ we have $\sum_{j \in J_{x} \cap J_{y}} \alpha_{j} \geq 1-2 \epsilon$. For convenience, we write $J_{x, y}$ for $J_{x} \cap J_{y}$. As sumPI $\left(f_{j}\right) \leq s$ there is a family of probability distributions $p^{j}$ such that whenever $f_{j}(x) \neq f_{j}(y)$

$$
\sum_{\substack{i \\ x_{i} \neq y_{i}}} \sqrt{p_{x}^{j}(i) p_{y}^{j}(i)} \geq 1 / s .
$$

Define $p_{x}(i)=\sum_{j \in J} \alpha_{j} p_{x}^{j}(i)$. Let $x, y$ be such that $f(x) \neq f(y)$. Then

$$
\begin{aligned}
\sum_{\substack{i \\
x_{i} \neq y_{i}}} \sqrt{p_{x}(i) p_{y}(i)} & =\sum_{\substack{i \\
x_{i} \neq y_{i}}} \sqrt{\sum_{j \in J} \alpha_{j} p_{x}^{j}(i)} \sqrt{\left.\sum_{j \in J} \alpha_{j} p_{y}^{j}(i)\right)} \\
& \geq \sum_{\substack{i \\
x_{i} \neq y_{i}}} \sqrt{\sum_{j \in J_{x, y}} \alpha_{j} p_{x}^{j}(i)} \sqrt{\sum_{j \in J_{x, y}} \alpha_{j} p_{y}^{j}(i)} \\
& \geq \sum_{\substack{i \\
x_{i} \neq y_{i}}} \sum_{j \in J_{x, y}} \sqrt{\alpha_{j} p_{x}^{j}(i)} \sqrt{\alpha_{j} p_{y}^{j}(i)} \\
& =\sum_{\substack{j \in J_{x, y}\\
}}\left(\alpha_{j} \sum_{\substack{i \\
x_{i} \neq y_{i}}} \sqrt{p_{x}^{j}(i) p_{y}^{j}(i)}\right) \\
& \frac{1-2 \epsilon}{s},
\end{aligned}
$$

where for the third step we have used the Cauchy-Schwarz Inequality.

This lemma immediately shows that the sumPI method can give lower bounds on probabilistic formula size.

Theorem 16 Let $S \subseteq\{0,1\}^{n}$ and $f: S \rightarrow\{0,1\}$. Then $\mathrm{L}^{\epsilon}(f) \geq((1-2 \epsilon) \operatorname{sumPI}(f))^{2}$ for any $\epsilon<1 / 2$.

Proof: Suppose that $\left\{f_{j}\right\}_{j \in J}$ gives an $\epsilon$-approximation to $f$. Using Lemma 15] in the contrapositive implies that there exists some $j \in J$ with $\operatorname{sumPI}\left(f_{j}\right) \geq(1-2 \epsilon) \operatorname{sumPI}(f)$. Theorem 14 then implies $\mathrm{L}\left(f_{j}\right) \geq((1-2 \epsilon) \text { sumPI }(f))^{2}$ which gives the statement of the theorem.

\section{Properties of sumPI and maxPI}

\subsection{Properties of sumPI}

Although in general sumPI gives weaker formula size lower bounds than maxPI, the measure sumPI has several nice properties which make it more convenient to use in practice.

The next lemma shows that the sumPI complexity behaves like most other complexity measures with respect to composition of functions: 
Lemma 17 Let $g_{1}, \ldots, g_{n}$ be functions from $S \subseteq \Sigma_{1}^{m}$ to $\Sigma_{2}$. For $x \in S$ define $g(x)=$ $\left(g_{1}(x), \ldots, g_{n}(x)\right)$. Define $S^{\prime}=\{g(x): x \in S\}$. Let $h$ be a function from $S^{\prime}$ to $T$. Assume that $\operatorname{sumPI}\left(g_{j}\right) \leq a$ for $1 \leq j \leq n$ and $\operatorname{sumPI}(h) \leq b$. Then for $f=h\left(g_{1}, \ldots, g_{n}\right)$ we have $\operatorname{sumPI}(f) \leq a b$.

Proof: Let $p$ be an optimal distribution function associated with $h$ and $p_{j}$ be an optimal distribution function associated with $g_{j}$. Define the distribution function

$$
q(x, i)=\sum_{j \in[n]} p(g(x), j) p_{j}(x, i)
$$

Assume that for $x, y \in S$ we have $f(x) \neq f(y)$. It is enough to show that

$$
\sum_{i: x_{i} \neq y_{i}} \sqrt{\sum_{j \in[n]} p(g(x), j) p_{j}(x, i)} \sqrt{\sum_{j \in[n]} p(g(y), j) p_{j}(y, i)} \geq \frac{1}{a b} .
$$

By Cauchy Schwarz, the left hand side of (4) is greater than or equal to

$$
\begin{array}{r}
\sum_{i: x_{i} \neq y_{i}} \sum_{j \in[n]} \sqrt{p(g(x), j) p_{j}(x, i)} \sqrt{p(g(y), j) p_{j}(y, i)} \\
=\sum_{j \in[n]}\left(\sqrt{p(g(x), j) p(g(y), j)} \sum_{i: x_{i} \neq y_{i}} \sqrt{p_{j}(x, i)} \sqrt{p_{j}(y, i)}\right) .
\end{array}
$$

As long as $g_{j}(x) \neq g_{j}(y)$, by the definition of $p_{j}$, we have $\sum_{i: x_{i} \neq y_{i}} \sqrt{p_{j}(x, i)} \sqrt{p_{j}(y, i)} \geq 1 / a$. Thus we can estimate the expression in (5) from below by:

$$
\frac{1}{a} \sum_{j: g_{j}(x) \neq g_{j}(y)} \sqrt{p(g(x), j) p(g(y), j)} .
$$

By the definition of $p$ we can estimate the sum (without the $1 / a$ coefficient) in the above expression from below by $1 / b$, which finishes the proof.

Another advantage of working with sumPI complexity is the following very powerful lemma of Ambainis Amb03 which makes it easy to lower bound the sumPI complexity of iterated functions.

Definition 18 Let $f:\{0,1\}^{n} \rightarrow\{0,1\}$ be any Boolean function. We define the dth iteration of $f$, written $f^{d}:\{0,1\}^{n^{d}} \rightarrow\{0,1\}$, inductively as $f^{1}(x)=f(x)$ and

$$
f^{d+1}(x)=f\left(f^{d}\left(x_{1}, \ldots, x_{n^{d}}, f^{d}\left(x_{n^{d}+1}, \ldots, x_{2 n^{d}}\right), \ldots, f^{d}\left(x_{(n-1) n^{d}+1}, \ldots, x_{n^{d+1}}\right)\right)\right.
$$

Lemma 19 (Ambainis) Let $f$ be any Boolean function and $f^{d}$ the dth iteration of $f$. Then $\operatorname{sumPI}\left(f^{d}\right) \geq(\operatorname{sumPI}(f))^{d}$.

Combining this with Lemma 17 we get:

Corollary 20 Let $f$ be any Boolean function and $f^{d}$ the dth iteration of $f$. Then $\operatorname{sumPI}\left(f^{d}\right)=$ $(\operatorname{sumPI}(f))^{d}$. 
Lemmas 8 and [17 together with the adversary argument lower bound for the Grover search Gro96, Amb02 imply that for total Boolean functions, the square root of the block sensitivity is a lower bound on the sumPI complexity. Hence, by $\mathrm{NS}^{2}, \mathrm{BBC}^{+} 01$ ]:

Lemma 21 For total Boolean functions the sumPI complexity is in polynomial relation with the various (deterministic, randomized, quantum) decision tree complexities and the Fourier degree of the function.

\subsection{Properties of maxPl}

One thing that makes sumPI so convenient to use is that it dualizes ŠS04. In this section we partially dualize the expression maxPI. The final expression remains a minimization problem, but we minimize over discrete index selection functions, instead of families of probability distributions, which makes it much more tractable.

Let $D_{i}, F$ be $|S| \times|S|$ Boolean matrices that satisfy $D_{i}[x, y]=1$ iff $x_{i} \neq y_{i}$ for $i \in[n]$, and $F[x, y]=1$ iff $f(x) \neq f(y)$. For a fixed family of probability distributions $p=\left\{p_{x}\right\}$, and for the expression

$$
\max _{\substack{x, y \\ f(x) \neq f(y)}} \frac{1}{\max _{i: x_{i} \neq y_{i}} \sqrt{p_{x}(i) p_{y}(i)}}
$$

let us define $P_{i}[x, y]=1$ if $i=\operatorname{argmax}_{i: x_{i} \neq y_{i}} \sqrt{p_{x}(i) p_{y}(i)}$ and 0 otherwise. (By definition argmax is the smallest argument for which the expression following the argmax attains its maximal value.) Then the denominator in Expression ([6) becomes equal to $\sum_{i: x_{i} \neq y_{i}} \sqrt{p(x, i) p(y, i)} P_{i}[x, y]$. The set of functions $\left\{P_{i}\right\}_{i}$ we call index selection function. If we replace the above system of $P_{i} \mathrm{~s}$ with any other choice of index selection function the value of $\sum_{i: x_{i} \neq y_{i}} \sqrt{p(x, i) p(y, i)} P_{i}[x, y]$ will not increase. Thus we can rewrite (6) as

$$
\max _{\substack{x, y \\ f(x) \neq f(y)}} \frac{1}{\max _{\left\{P_{i}\right\}_{i}} \quad \sum_{i: x_{i} \neq y_{i}} \sqrt{p(x, i) p(y, i)} P_{i}[x, y]} .
$$

Here $P_{i}[x, y]$ runs through all index selection functions, i.e. all Boolean valued functions with the properties that for every $x, y \in S$ :

1. $\sum_{i} P_{i}[x, y]=F[x, y]$ (informally: we select a unique index if $f(x) \neq f(y)$ );

2. $P_{i}[x, y] \leq D_{i}[x, y]$ (informally: the index we select is an $i$ such that $x_{i} \neq y_{i}$ ).

Thus:

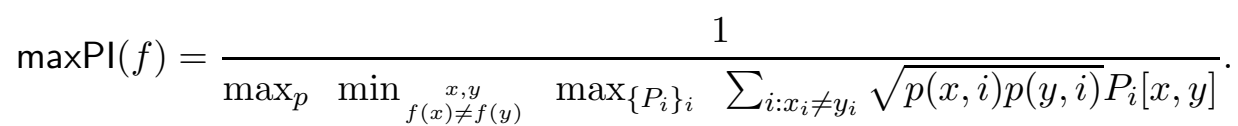

Notice that in (77) the minimum is interchangeable with the second maximum. The reason for this is that for a fixed $p$ there is a fixed $\left\{P_{i}[x, y]\right\}_{i}$ system that maximizes $\sum_{i: x_{i} \neq y_{i}} \sqrt{p(x, i) p(y, i)} P_{i}[x, y]$ for all $x, y: f(x) \neq f(y)$. Thus:

$$
\max \mathrm{PI}(f)=\frac{1}{\max _{\left\{P_{i}\right\}_{i}} \max _{p} \min _{\substack{x, y \\ f(x) \neq f(y)}} \sum_{i: x_{i} \neq y_{i}} \sqrt{p(x, i) p(y, i)} P_{i}[x, y]} .
$$


Following the proof of the main theorem of Špalek and Szegedy we can create the semidefinite version of the above expression. The difference here, however, is that we have to treat $\left\{P_{i}\right\}_{i}$ (the index selection functions) as a "parameter" of the semidefinite system over which we have to maximize. Unfortunately it also appears in the final expression.

Semidefinite version of maxPI: For fixed $\left\{P_{i}\right\}_{i}$ let $\mu_{\max }^{\prime}$ be the solution of the following semidefinite program:

$$
\begin{aligned}
\operatorname{maximize} & \mu^{\prime} \\
\text { subject to } \quad(\forall i) \quad R_{i} & \succeq 0, \\
\sum_{i} R_{i} \circ I & =I, \\
\sum_{i} R_{i} \circ P_{i} & \geq \mu^{\prime} F .
\end{aligned}
$$

Define $\mu_{\max }$ as the maximum of $\mu_{\max }^{\prime}$, where $P_{i}(1 \leq i \leq n)$ run through all Boolean matrices such that $\sum_{i} P_{i}=F$ and for every $1 \leq i \leq n$ : $P_{i} \leq D_{i}$ entry-wise. Then maxPI $=1 / \mu_{\max }$.

We can dualize the above program and simplify it in same way as was done in Špalek and Szegedy for the case of sumPI with the only change that $D_{i}$ needs to be replaced with $P_{i}$, and that we have to minimize over all choices of $\left\{P_{i}\right\}_{i}$. We obtain:

Spectral adversary version of maxPI: Let $\Gamma$ denote an arbitrary $|S| \times|S|$ non-negative symmetric matrix that satisfies $\Gamma[x, y]=0$ whenever $f(x)=f(y)$. Then

$$
\max \mathrm{PI}(f)=\min _{\left\{P_{i}\right\}_{i}} \max _{\Gamma} \frac{\lambda(\Gamma)}{\max _{i} \lambda\left(\Gamma \circ P_{i}\right)},
$$

where $\left\{P_{i}\right\}_{i}$ runs through all the index selection functions.

\section{Comparison Among Methods}

\subsection{Khrapchenko's method}

One of the most general techniques available for showing formula size lower bounds is Khrapchenko's method Khr71, originally used to give a tight $\Omega\left(n^{2}\right)$ lower bound for the parity function. This method considers a bipartite graph with color classes the positive and negative instances of a function $f$, and edges between pairs which differ in exactly one bit. The bound given is the product of the average degree of the color classes.

Theorem 22 (Khrapchenko) Let $S \subset\{0,1\}^{n}$ and $f: S \rightarrow\{0,1\}$. Let $A \subseteq f^{-1}(0)$ and $B \subseteq$ $f^{-1}(1)$. Let $C=\left\{(x, y) \in A \times B: d_{H}(x, y)=1\right\}$. Then $\mathrm{L}(f) \geq \frac{|C|^{2}}{|A||B|}$.

Khrapchenko's method can easily be seen as a special case of the probability scheme, where we assign positive probabilities only to those pairs $(x, y)$ of positive and negative instances which differ in exactly one bit, and use uniform distributions everywhere. More formally, letting $A, B, C$ be as in the statement of the theorem, we set up our probability distributions as follows:

- let $p_{A}(x)=1 /|A|$ for all $x \in A$ and $p_{A}(x)=0$ otherwise

- let $p_{B}(x)=1 /|B|$ for all $x \in B$ and $p_{B}(x)=0$ otherwise

- let $q(x, y)=1 /|C|$ for all $(x, y) \in C$ and $q(x, y)=0$ otherwise 
- let $p_{x, i}(y)=1$ if $(x, y) \in C$ and $x_{i} \neq y_{i}$ and 0 otherwise. Note that this is a probability distribution as for every $x$ there is only one $y$ such that $(x, y) \in C$ and $x_{i} \neq y_{i}$.

Whenever $q(x, y) \neq 0$ then $p_{A}(x), p_{B}(x), p_{x, i}^{\prime}(y), p_{y, i}^{\prime}(x)$ are nonzero for the $i$ such that $x_{i} \neq y_{i}$, thus these distributions form a valid probability scheme and by Theorem 14 we obtain

$$
\mathrm{L}(f) \geq \min _{\substack{x, y, i \\ f(x) \neq f(y), x_{i} \neq y_{i}}} \frac{p_{A}(x) p_{B}(y) p_{x, i}^{\prime}(y) p_{y, i}^{\prime}(x)}{q(x, y)}=\frac{|C|^{2}}{|A||B|}
$$

\subsection{The Koutsoupias Bound}

Koutsoupias Kou93 extended Khrapchenko's technique with a spectral version. The weights are always 1 for pairs of inputs with different function values that differ in one variable, and 0 everywhere else.

Theorem 23 (Koutsoupias) Let $f:\{0,1\}^{n} \rightarrow\{0,1\}$, and let $A \subseteq f^{-1}(0)$, and $B \subseteq f^{-1}(1)$. Let $C=\left\{(x, y) \in A \times B: d_{H}(x, y)=1\right\}$. Let $Q$ be a $|B| \times|A|$ matrix $Q[x, y]=C(x, y)$ where $C$ is identified with its characteristic function. Then $\mathrm{L}(f) \geq \lambda\left(Q Q^{T}\right)$, where $\lambda$ denotes the largest eigenvalue.

Proof: In order to reprove the Koutsoupias bound we use the spectral method. Let $Q$ be the matrix as in the statement of the theorem whose elements are indexed by $A \times B$. On $(A \cup B) \times(A \cup B)$ we create the adversary matrix

$$
\Gamma=\left(\begin{array}{ll}
0 & Q \\
Q^{T} & 0
\end{array}\right)
$$

It is a well known fact that the spectral norm of $\Gamma$ is exactly $\sqrt{\lambda\left(Q Q^{T}\right)}$. It is also easy to see that $\Gamma_{i}$ is an adjacency matrix of the matching $M_{i}=\{(x, y) \in A \times B: x$ and $y$ differ only in coordinate $i\}$, if we view $M_{i}$ as a graph on the vertex set $A \cup B$. Hence $\lambda\left(\Gamma_{i}\right)=1$ if $M_{i}$ is non-empty, and 0 if $M_{i}$ is empty. Therefore:

$$
\frac{\lambda(\Gamma)}{\max _{i} \lambda\left(\Gamma_{i}\right)}=\sqrt{\lambda\left(Q Q^{T}\right)} / 1=\sqrt{\lambda\left(Q Q^{T}\right)}
$$

Koutsoupias gives an example of a function where his method is better by a multiplicative factor of 2 than the best possible bound that can be obtained with Khrapchenko's technique. In Section 7 we show a function on $N$ variables where the Khrapchenko method gives a lower bound of no more than $N$ (a trivial lower bound as the function depends on all of its variables) and the sumPI method gives a lower bound of $\Omega\left(N^{1.3}\right)$. Our example function is the same as the one Ambainis used to show a separation between the adversary method and the polynomial method Amb03.

\subsection{Håstad's method}

The shrinkage exponent of Boolean formulae is the least upper bound $\gamma$ such that subject to a random restriction where each variable is left free with probability $p$, Boolean formulae shrink from size $L$ to expected size $p^{\gamma} L$. To prove that the shrinkage exponent of Boolean formulae is 2 , Håstad proves an intermediate lemma, which gives a lower bound on formula size that depends on the 
probability that restrictions of a certain form occur. Håstad then goes on to prove a lower bound of $n^{3}$ (up to logarithmic factors) for Andreev's function And87. He proves that the intermediate method is a generalization of Khrapchenko's method; we prove that Håstad's method is in turn a special case of sumPI. Since Håstad's method uses random restrictions, which at first glance seems completely different from adversary methods, it comes as a surprise that it is in fact a special case of our techniques.

Definition 24 Let $f$ be a Boolean function over $n$ variables.

1. A restriction is a string in $\{0,1, \star\}^{n}$ where $\star$ means the variable is left free, and 0 or 1 mean the variable is set to the constant 0 or 1 , respectively.

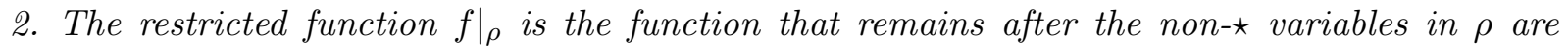
fixed.

3. $R_{p}$ is the distribution on random restrictions to the variables of $f$ obtained by setting each variable, independently, to $\star$ with probability $p$, and to 0 or 1 each with probability $\frac{(1-p)}{2}$.

4. A filter $\Delta$ is a set of restrictions which has the property that if $\rho \in \Delta$, then every $\rho^{\prime}$ obtained by fixing one of the $\star s$ to a constant is also in $\Delta$.

5. When $p$ is known from the context, and for any event $E$, and any filter $\Delta$, we write $\operatorname{Pr}[E \mid \Delta]$ to mean $\operatorname{Pr}_{\rho \in R_{p}}[E \mid \rho \in \Delta]$.

Theorem 25 ([Hås98], Lemma 4.1) Let $f:\{0,1\}^{n} \rightarrow\{0,1\}$. Let $A$ be the event that a random restriction in $R_{p}$ reduces $f$ to the constant $0, B$ be the event that a random restriction in $R_{p}$ reduces $f$ to the constant 1 , and let $C$ be the event that a random restriction $\rho \in R_{p}$ is such that $\left.f\right|_{\rho}$ is a single literal. Then

$$
\mathrm{L}(f) \geq \frac{\operatorname{Pr}[C \mid \Delta]^{2}}{\operatorname{Pr}[A \mid \Delta] \operatorname{Pr}[B \mid \Delta]}\left(\frac{1-p}{2 p}\right)^{2}
$$

Proof: We show that the theorem follows from the probability scheme (Definition 4). In this proof we only consider restrictions obtained from $R_{p}$ that are in the filter $\Delta$. We also abuse notation and use $A$ and $B$ to mean the sets of restrictions in $\Delta$ which contribute with non-zero probability to the events $A$ and $B$ respectively.

Implicit in Håstad's proof is the following relation between restrictions in $A$ and $B$. For every $\rho \in C,\left.f\right|_{\rho}$ reduces to a single literal, that is, for every $\rho \in C$, there is an $i$ such that $\left.f\right|_{\rho}=x_{i}$ (or $\neg x_{i}$ if the variable is negated). Define $\rho^{b}$ to be $\rho$ where $x_{i}$ is set to $b$, for $b \in\{0,1\}$ (set $x_{i}$ to $1-b$ if the variable is negated). To fit into the framework of the probability scheme, let $\overline{\rho^{b}}$ be $\rho^{b}$ where all remaining $\star$ s are set to 1 . This doesn't change the value of the function, because it is already constant on $\rho^{b}$. Then we say that $\overline{\rho^{0}}, \overline{\rho^{1}}$ are in the relation.

We set $p_{A}(\sigma)=\frac{\operatorname{Pr}[\sigma]}{\operatorname{Pr}[A \mid \Delta]}$ for any $\sigma \in A$, and $p_{B}(\tau)=\frac{\operatorname{Pr}[\tau]}{\operatorname{Pr}[B \mid \Delta]}$ for any $\tau \in B$, and for every pair $\overline{\rho^{0}}, \overline{\rho^{1}}$ in the relation, where $\rho \in C,\left.f\right|_{\rho}=x_{i}$ or $\neg x_{i}$, let

$$
\begin{aligned}
& p \frac{\bar{\rho}^{0}, i}{\prime}\left(\overline{\rho^{1}}\right)=1 \\
& p_{\overline{\rho^{1}}, i}^{\prime}\left(\overline{\rho^{0}}\right)=1 \\
& q\left(\overline{\rho^{0}}, \overline{\rho^{1}}\right)=\frac{\operatorname{Pr}[\rho]}{\operatorname{Pr}[C \mid \Delta]}
\end{aligned}
$$


The probabilities are 0 on all other inputs. We can easily verify that the probabilities sum to 1 . For $p^{\prime}$, notice that the Hamming distance between $\overline{\rho^{0}}$ and $\overline{\rho^{1}}$ is 1 , so when $\overline{\rho^{b}}$ and $i$ are fixed, there is only a single $\overline{\rho^{1-b}}$ with probability 1 .

By Theorems 6] and [14.

$$
\begin{aligned}
\mathrm{L}(f) & \geq \frac{p_{A}(x) p_{B}(y) p_{y, i}^{\prime}(x) p_{x, i}^{\prime}(y)}{q(x, y)^{2}} \\
& =\frac{\operatorname{Pr}\left[\rho^{0}\right]}{\operatorname{Pr}[A \mid \Delta]} \frac{\operatorname{Pr}\left[\rho^{1}\right]}{\operatorname{Pr}[B \mid \Delta]}\left(\frac{\operatorname{Pr}[C \mid \Delta]}{\operatorname{Pr}[\rho]}\right)^{2}
\end{aligned}
$$

Finally, as Håstad remarks (and is easy to see), $\operatorname{Pr}[\rho]=\frac{2 p}{1-p} \operatorname{Pr}\left[\rho^{b}\right]$.

Remark Håstad actually defines $\left.f\right|_{\rho}$ to be the result of reducing the formula for $f$ (not the function) by applying a sequence of reduction rules, for each restricted variable. So there is a subtlety here about whether $\left.f\right|_{\rho}$ denotes the reduced formula, or the reduced function, and the probabilities might be different if we are in one setting or the other. However both in his proof and ours, the only thing that is used about the reduction is that if the formula or function reduces to a single literal, then fixing this literal to 0 or to 1 reduces the function to a constant. Therefore, both proofs go through for both settings.

\section{$6 \quad$ Limitations}

The limitations of the adversary method are well known Amb02, LM04, Sze03, Zha04, SS04. Špalek and Szegedy, in unifying the adversary methods, also give the most elegant proof of their collective limitation. The same proof also shows the same limitations hold for the maxPI measure.

Lemma 26 Let $f:\{0,1\}^{n} \rightarrow\{0,1\}$ be any partial or total Boolean function. If $f$ is total (respectively, partial) then $\operatorname{maxPI}(f) \leq \sqrt{C_{0}(f) C_{1}(f)}$ (respectively, $\min \left\{n C_{0}(f), n C_{1}(f)\right\}$ ).

Proof: Assume that $f$ is total. Take $x, y$ such that $f(x)=0$ and $f(y)=1$. We choose any 0-certificate $B_{0}$ for $x$ and any 1-certificate $B_{1}$ for $y$ and let $p_{x}(i)=1 / C_{0}(f)$ for all $i \in B_{0}$ and $p_{y}(i)=1 / C_{1}(f)$ for all $i \in B_{1}$. As $f$ is total, we have $B_{0} \cap B_{1} \neq \emptyset$, thus let $j \in B_{0} \cap B_{1}$. For this $j$ we have $p_{x}(j) p_{y}(j) \geq 1 /\left(C_{0}(f) C_{1}(f)\right)$, thus $\min _{i} 1 / p_{x}(i) p_{y}(i) \geq C_{0}(f) C_{1}(f)$.

The case where $f$ is partial follows similarly. As we no longer know that $B_{0} \cap B_{1} \neq \emptyset$, we put a uniform distribution over a 0 -certificate of $x$ and the uniform distribution over $[n]$ on $y$ or vice versa.

As Khrapchenko's method only considers pairs of positive and negative instances with Hamming distance 1 , its limitations are even more severe than maxPI.

Lemma 27 The bound given by the Khrapchenko method of Theorem 20 for a function $f$ is at most $s^{0}(f) s^{1}(f) \leq s^{2}(f)$.

Proof: Let $A \subseteq f^{-1}(0)$ and $B \subseteq f^{-1}(1)$ and $C$ be the set of $(x, y)$ where $(x, y) \in A \times B$ and $x$ and $y$ differ in exactly one coordinate. The maximum degree of any element $x \in A$ is $s^{0}(f)$ and similarly the maximum degree of any element $y \in B$ is $s^{1}(f)$. Thus as the Khrapchenko bound is 
the product of average degrees in $A$ and $B$ it is bounded by $s^{0}(f) s^{1}(f)$.

Schürfeld Sch83] proves a weaker version of this lemma, showing that Khrapchenko cannot prove bounds greater than $C_{0}(f) C_{1}(f)$.

Besides the certificate complexity barrier, another serious limitation of the sumPI method occurs for partial functions where every positive input is far in Hamming distance from every negative input. Thus for example, if for any pair $x, y$ where $f(x)=1$ and $f(y)=0$ we have $d_{H}(x, y) \geq \epsilon n$, then by putting the uniform distribution over all input bits it follows that $\operatorname{sumPI}(f) \leq 1 / \epsilon$. The measure maxPI does not face this limitation as there we still only have one term in the denominator.

Following this line of thinking, we can give an example of a partial function $f$ where $\max \mathrm{PI}(f) \gg$ sumPI $(f)$. Such an example is the Collision problem (see Section [7.3), as here any positive and negative input must differ on at least $n / 2$ positions. Another family of examples comes from property testing, where the promise is exactly what we describe, that is, that the input either has some property, or that it is $\epsilon$-far from having the property.

\section{Applications}

The quantum adversary argument has been used to prove lower bounds for a variety of problems. Naturally, all of these lower bounds carry over to formula size lower bounds. In this section we present some new lower bounds, in order to highlight the strenghts and weaknesses of maxPI and sumPI.

\subsection{Recursive Majorities}

As an example of applying sumPI, we look at the recursive majority of three function. We let R-MAJ $J_{3}^{h}:\{0,1\}^{3^{h}} \rightarrow\{0,1\}$ be the function computed by a complete ternary tree of depth $h$ where every internal node is labeled by a majority gate and the input is given at the leaves.

Recursive majority of three has been studied before in various contexts. It is a monotone function which is very sensitive to noise [M003, making it useful for hardness amplification in NP O'D02. Jayram, Kumar, and Sivakumar JKS03 give nontrivial lower and upper bounds on the randomized decision tree complexity of recursive majority of three. They show a lower bound of $(7 / 3)^{h}$ on the randomized decision tree complexity. As far as we know, the quantum query complexity of recursive majority of three has not yet been investigated. We show a lower bound of $2^{h}$ on the quantum query complexity.

Lemma 28 sumPI $\left(\mathrm{R}-\mathrm{MAJ}_{3}^{h}\right)=\operatorname{maxPI}\left(\mathrm{R}-\mathrm{MAJ}_{3}^{h}\right)=2^{h}$

Proof: To see that $\operatorname{maxPI}\left(\mathrm{R}-\mathrm{MAJ} J_{3}^{h}\right) \leq 2^{h}$, observe that $C_{0}\left(\mathrm{R}-\mathrm{MAJ}{ }_{3}^{h}\right)=C_{1}\left(\mathrm{R}-\mathrm{MAJ}{ }_{3}^{h}\right)=2^{h}$. The result then follows from Lemma 26.

We now turn to the lower bound. We will first show a lower bound for $\mathrm{R}-\mathrm{MAJ}_{3}^{1}$, the majority of three function, and then apply Lemma 19. Consider the following table, where the rows are indexed by negative instances $x$, the columns by positive instances $y$, and 1's indicate when $d_{H}(x, y)=1$.

\begin{tabular}{c|ccc} 
& 110 & 101 & 011 \\
\hline 001 & 0 & 1 & 1 \\
010 & 1 & 0 & 1 \\
100 & 1 & 1 & 0
\end{tabular}


Interpreting this table as the adjacency matrix of a graph, it is clear that every vertex has degree 2. Thus Khrapchenko's method gives a bound of 4 for the base function. By Theorem 22 we have sumPI $\left(\mathrm{R}-\mathrm{MAJ}_{3}^{1}\right) \geq 2$. Now applying Lemma 19 gives the lemma.

From Lemma 28 we immediately obtain quantum query complexity and formula size lower bounds:

Theorem 29 Let $\mathrm{R}-\mathrm{MAJ}_{3}^{h}$ be the recursive majority of three function of height $h$. Then $Q_{\epsilon}\left(\mathrm{R}-\mathrm{MAJ}_{3}^{h}\right) \geq(1-2 \sqrt{\epsilon(1-\epsilon)}) 2^{h}$ and $\mathrm{L}^{\epsilon}\left(\mathrm{R}-\mathrm{MAJ}_{3}^{h}\right) \geq(1-2 \epsilon) 4^{h}$

The best upper bound on the formula size of $\mathrm{R}-\mathrm{MAJ}_{3}^{h}$ is $5^{h}$. For this bound, we will use the following simple proposition about the formula size of iterated functions.

Proposition 30 Let $S \subseteq\{0,1\}^{n}$ and $f: S \rightarrow\{0,1\}$. If $\mathrm{L}(f) \leq s$ then $\mathrm{L}\left(f^{d}\right) \leq s^{d}$, where $f^{d}$ is the dth iteration of $f$.

Proposition $31 \mathrm{~L}\left(\mathrm{R}-\mathrm{MAJ}_{3}^{h}\right) \leq 5^{h}$

Proof: The formula $\left(x_{1} \wedge x_{2}\right) \vee\left(\left(x_{1} \vee x_{2}\right) \wedge x_{3}\right)$ computes R-MAJ ${ }_{3}^{1}$ and has 5 leaves. Using Proposition 30] gives $\mathrm{L}\left(\mathrm{R}-\mathrm{MAJ}_{3}^{h}\right) \leq 5^{h}$.

\subsection{Ambainis' function}

We define a function $\mathrm{f}_{\mathrm{A}}:\{0,1\}^{4} \rightarrow\{0,1\}$ after Ambainis Amb03. This function evaluates to 1 on the following values: $0000,0001,0011,0111,1111,1110,1100,1000$. That is, $f(x)=1$ when $x_{1} \leq x_{2} \leq x_{3} \leq x_{4}$ or $x_{1} \geq x_{2} \geq x_{3} \geq x_{4}$. To obtain this formulation from Ambainis' original definition, exchange $x_{1}$ and $x_{3}$, and take the negation of the resulting function. There are a few things to notice about this function. The sensitivity of $f_{A}$ is 2 on every input. Also on an input $x=x_{1} x_{2} x_{3} x_{4}$ the value of $\mathrm{f}_{\mathrm{A}}(x)$ changes if both bits sensitive to $x$ are flipped, and if both bits insensitive for $x$ are flipped.

We will be looking at iterations of the base function $\mathrm{f}_{\mathrm{A}}$ as in definition 18. Notice that the sensitivity of $\mathrm{f}_{\mathrm{A}}{ }^{d}$ is $2^{d}$ on every input $x \in\{0,1\}^{4^{d}}$.

Lemma 32 sumPI $\left(\mathrm{f}_{\mathrm{A}}{ }^{d}\right)=2.5^{d}$.

Proof: Ambainis has already shown that sumPI $\left(\mathrm{f}_{\mathrm{A}}{ }^{d}\right) \geq 2.5^{d}$ Amb03.

We now show the upper bound. We will show an upper bound for the base function $\mathrm{f}_{\mathrm{A}}$ and then use the composition Lemma 17. Every input $x_{1} x_{2} x_{3} x_{4}$ has two sensitive variables and two insensitive variables. For any $x \in\{0,1\}^{4}$ we set $p_{x}(i)=2 / 5$ if $i$ is sensitive for $x$ and $p_{x}(i)=1 / 10$ if $i$ is insensitive for $x$. The claim follows from the following observation: for any $x, y \in\{0,1\}^{4}$ such that $f(x) \neq f(y)$ at least one of the following holds

- $x$ and $y$ differ on a position $i$ which is sensitive for both $x$ and $y$. Thus $\sum_{i} \sqrt{p_{x}(i) p_{y}(i)} \geq 2 / 5$

- $x$ and $y$ differ on at least 2 positions, each of these positions being sensitive for at least one of $x, y$. Thus $\sum_{i} \sqrt{p_{x}(i) p_{y}(i)} \geq 2 \sqrt{1 / 25}=2 / 5$ 
This lemma gives us a bound of $6.25^{d} \approx N^{1.32}$ on the formula size of $\mathrm{f}_{\mathrm{A}}{ }^{d}$. Since the sensitivity of $\mathrm{f}_{\mathrm{A}}{ }^{d}$ is $2^{d}$, by Lemma 27. the best bound provable by Khrapchenko is $4^{d}=N$.

It is natural to ask how tight this formula size bound is. The best upper bound we can show on the formula size of $\mathrm{f}_{\mathrm{A}}{ }^{d}$ is $12^{d}$.

Proposition $33 \mathrm{~L}\left(\mathrm{f}_{\mathrm{A}}{ }^{d}\right) \leq 12^{d}$

Proof: We show that the basis function $\mathrm{f}_{\mathrm{A}}$ has a formula size 12 and then the proposition follows from Proposition [30. The basis function can be written as $\mathrm{f}_{\mathrm{A}}(x)=\left(x_{1} \leq x_{2} \leq x_{3} \leq x_{4}\right) \vee\left(x_{1} \geq\right.$ $\left.x_{2} \geq x_{3} \geq x_{4}\right)$. We can write $x_{i} \leq x_{j}$ as $\neg x_{i} \vee x_{j}$ which has 2 leaves. By taking the AND of these comparisons we obtain a formula of size 6 for the expression $x_{1} \leq x_{2} \leq x_{3} \leq x_{4}$, and similarly for $x_{1} \geq x_{2} \geq x_{3} \geq x_{4}$, and thus a formula of size 12 for $\mathrm{f}_{\mathrm{A}}$.

\subsection{Collision Problem}

In this section we look at the collision problem. This is a promise problem, where for an alphabet $\Sigma$ the inputs $x=x_{1} x_{2} \ldots x_{n} \in \Sigma^{n}$ satisfy one of the following conditions:

- All $x_{i}$ are different

- For each $i$ there exists exactly one $j \neq i$ such that $x_{i}=x_{j}$.

Those inputs satisfying the first condition are positive inputs and those satisfying the second condition are negative. An optimal lower bound for the quantum query complexity of $\Omega\left(n^{1 / 3}\right)$ has been given by Aaronson and Shi AS04. We now show that the quantum adversary method cannot give better than a constant bound for this problem.

Lemma 34 sumPI $\left(f_{C}\right) \leq 2$

Proof: We demonstrate a set of probability distributions $p_{x}$ such that for any positive instance $x$ and negative instance $y$ we have

$$
\sum_{\substack{i \\ x_{i} \neq y_{i}}} \sqrt{p_{x}(i)} \sqrt{p_{y}(i)} \geq 1 / 2
$$

The upper bound then follows.

Our probability distribution is very simple: for every $x$, let $p_{x}(i)$ be the uniform distribution over $[n]$. Any positive and negative instance must disagree in at least $n / 2$ positions, thus

$$
\sum_{\substack{i \\ x_{i} \neq y_{i}}} \sqrt{p_{x}(i)} \sqrt{p_{y}(i)} \geq \frac{n}{2} \sqrt{\frac{1}{n} \frac{1}{n}}=\frac{1}{2} .
$$

On the other hand, $\operatorname{maxPI}\left(\mathrm{f}_{\mathrm{C}}\right) \geq \sqrt{n / 2}$. As there is an upper bound for the collision problem of $O\left(n^{1 / 3}\right)$ by Brassard, Høyer, Tapp BHT97, this also shows that in general maxPI $(f)$ is not a lower bound on the quantum query complexity of $f$. 


\section{Lemma $35 \operatorname{maxPI}\left(f_{\mathrm{C}}\right)=\Theta(\sqrt{n})$}

Proof: For the upper bound: On every positive instance $x$, where all $x_{i}$ are different, we put the uniform distribution over $i \in[n]$; for a negative instance $y$ we put probability $1 / 2$ on the first position, and probability $1 / 2$ on the position $j$ such that $y_{1}=y_{j}$. As $y_{1}=y_{j}$, any positive instance $x$ must differ from $y$ on position 1 or position $j$ (or both). Thus $\max _{i, x_{i} \neq y_{i}} p_{x}(i) p_{y}(i) \geq 1 / 2 n$ and $\max \mathrm{PI}\left(\mathrm{f}_{\mathrm{C}}\right) \leq \sqrt{2 n}$.

Now for the lower bound. Fix a set of probability distributions $p_{x}$. Let $x$ be any positive instance. There must be at least $n / 2$ positions $i$ satisfying $p_{x}(i) \leq 2 / n$. Call this set of positions $I$. Now consider a negative instance $y$ of where $y_{j}=x_{j}$ for all $j \notin I$, and $y$ is assigned values in $I$ in an arbitrary way so as to make it a negative instance. For this pair $x, y$ we have $\max _{i} \sqrt{p_{x}(i)} \sqrt{p_{y}(i)} \leq$ $\sqrt{2 / n}$, thus $\max \mathrm{PI}\left(\mathrm{f}_{\mathrm{C}}\right) \geq \sqrt{n / 2}$.

The following table summarizes the bounds from this section.

\begin{tabular}{|c|c|c|c|c|c|c|}
\hline Function & Input size & sumPI & $\overline{Q_{\epsilon}}$ & $\operatorname{maxPI}$ & $\mathrm{L}$ & $s^{0} s^{1}$ \\
\hline$\overline{\mathrm{R}-\mathrm{MAJ}_{3}^{h}}$ & $\bar{~} N=3^{h}$ & $\begin{aligned} & 2^{h} \\
\approx & N^{0.63}\end{aligned}$ & $\overline{\Omega\left(\Omega\left(N^{0.63}\right)\right.}$ & $\bar{c} N^{0.63}$ & $\begin{array}{l}\Omega\left(N^{1.26}\right), \\
O\left(N^{1.46}\right)\end{array}$ & $\bar{~} N^{1.26}$ \\
\hline $\mathrm{f}_{\mathrm{A}}{ }^{h}$ & $N=4^{h}$ & $\begin{aligned} & 2.5^{h} \\
\approx & N^{0.66}\end{aligned}$ & $\begin{array}{r}\Omega\left(N^{0.66}\right) \\
\text { Amb03 }\end{array}$ & $\begin{array}{c}\leq 3^{h} \\
\approx N^{0.79}\end{array}$ & $\begin{array}{l}\Omega\left(N^{1.32}\right), \\
O\left(N^{1.79}\right)\end{array}$ & $N$ \\
\hline $\mathrm{f}_{\mathrm{C}}$ & $N$ & 2 & $\Theta\left(N^{1 / 3}\right)$ & $\Theta(\sqrt{N})$ & $N$ & $\perp$ \\
\hline
\end{tabular}

\section{Conclusions and Open Problems}

Our new formula size lower bound techniques subsume many previous techniques, and for some functions they are provably better. A significant part of our intuition comes from quantum query complexity and Kolmogorov complexity. Measures sumPI and maxPI have many interesting properties and they connect different complexities such as quantum query complexity, classical formula size, classical probabilistic formula size and circuit depth.

An outstanding open problem is whether the square of the quantum query complexity lower bounds the formula size. Another is that we do not know a nice dual expression for maxPI, and it does not seem to be a natural property in the sense of Razborov and Rudich. Thus the study of maxPI may lead us to a better understanding of complexity measures that themselves are hard to compute. We could reprove a key lemma of Håstad that leads to the best current formula size lower bound and we are hopeful that our techniques eventually will lead to improvements of the bounds in Hås98.

\section{Acknowledgments}

We would like to thank Frédéric Magniez, Robert Špalek, and Ronald de Wolf for helpful discussions. We also wish to thank Ryan O'Donnell for suggesting to look at the recursive majority of three function. 


\section{References}

[Aar04] S. Aaronson. Lower bounds for local search by quantum arguments lower bounds for local search by quantum arguments. In Proceedings of the thirty-sixth annual ACM symposium on Theory of computing, pages 465-474, 2004.

[Amb02] A. Ambainis. Quantum lower bounds by quantum arguments. Journal of Computer and System Sciences, 64:750-767, 2002.

[Amb03] A. Ambainis. Polynomial degree vs. quantum query complexity. In Proceedings of 44th IEEE Symposium on Foundations of Computer Science, pages 230-239, 2003.

[And87] A. E. Andreev. On a method for obtaining more than quadratic effective lower bounds for the complexity of П-schemes. Moscow Univ. Math. Bull., 42(1):63-66, 1987.

[AS04] S. Aaronson and Y. Shi. Quantum lower bounds for the collision and the element distinctness problems. Journal of the ACM, 51(4):595 - 605, 2004.

[BBBV97] C.H. Bennett, E. Bernstein, G. Brassard, and U. Vazirani. Strengths and weaknesses of quantum computing. SIAM Journl on Computing, 26:1510-1523, 1997.

$\left[\mathrm{BBC}^{+} 01\right]$ R. Beals, H. Buhrman, R. Cleve, M. Mosca, and R. de Wolf. Quantum lower bounds by polynomials. Journal of the ACM, 48(4):778-797, 2001.

[BHT97] G. Brassard, P. Høyer, and A. Tapp. Quantum algorithm for the collision problem. ACM SIGACT News (Cryptology column), 28:14-19, 1997.

[Bop89] R. Boppana. Amplification of probabilistic boolean formulas. Advances in Computing Research, 5(4):27-45, 1989.

[BSS03] H. Barnum, M. Saks, and M. Szegedy. Quantum decision trees and semidefinite programming. In Proceedings of the 18th IEEE Conference on Computational Complexity, pages 179-193, 2003.

[BW02] H. Buhrman and R. de Wolf. Complexity measures and decision tree complexity: A survey. Theoretical Computer Science, 288:21-43, 2002.

[DZ97] M. Dubiner and U. Zwick. Amplification by read-once formulas. SIAM J. Comput., 26(1):15-38, 1997.

[Gro96] L. Grover. A fast quantum mechanical algorithm for database search. In Proceedings of 28th ACM Symposium on Theory of Computing, pages 212-219, 1996.

[Hås98] J. Håstad. he shrinkage exponent of de morgan formulae is 2. SIAM Journal on Computing, 2719(1):48-64, 1998.

[IM02] K. Iwama and H. Morizumi. An explicit lower bound of $5 n-o(n)$ for boolean circuits. In Proceedings of the 27th International Symposium on Mathematical Foundations of Computer Science, pages 353-364, 2002. 
[JKS03] T. Jayram, R. Kumar, and D. Sivakumar. Two applications of information complexity. In Proceedings of the 35th ACM Symposium on the Theory of Computing, pages 673-682, 2003.

[Khr71] V.M. Khrapchenko. Complexity of the realization of a linear function in the case of П-circuits. Math. Notes Acad. Sciences, 9:21-23, 1971.

[Kla04] H. Klauck. One-way communication complexity and the neciporuk lower bound on formula size. Technical Report 0111062, cs.CC arXiv, 2004.

[Kou93] E. Koutsoupias. Improvements on Khrapchenko's theorem. Theoretical Computer Science, 116(2):399-403, 1993.

[KW88] M. Karchmer and A. Wigderson. Monotone connectivity circuits require superlogarithmic depth. In Proceedings of the 20th STOC, pages 539-550, 1988.

[KW03] I. Kerenidis and R. de Wolf. Exponential lower bound for 2-query locally decodable codes via a quantum argument. In Proceedings of the thirty-fifth annual ACM symposium on Theory of computing, pages 106-115, 2003.

[LM04] S. Laplante and F. Magniez. Lower bounds for randomized and quantum query complexity using kolmogorov arguments. In Proceedings of the Nineteenth Annual IEEE Conference on Computational Complexity, pages 294-304, 2004.

[LR01] O. Lachish and R. Raz. Explicit lower bound of 4.5n - o(n) for boolean circuits. In Proceedings of the thirty-third annual ACM symposium on Theory of computing, pages 399-408, 2001.

[LV97] M. Li and P. Vitányi. An introduction to Kolmogorov complexity and its applications. In Graduate Texts in Computer Science. Springer, 1997. Second edition.

[MO03] E. Mossell and R. O'Donnell. On the noise sensitivity of monotone functions. Random Structures and Algorithms, 23(3):333-350, 2003.

[NC00] M. Nielsen and I. Chuang. Quantum Computation and Quantum Information. Cambridge University Press, 2000.

[NS94] N. Nisan and M. Szegedy. On the degree of boolean functions as real polynomials. Computational Complexity, 4:301-313, 1994.

[O'D02] R. O'Donnell. Hardness amplification within NP. In Proceedings of the 34th ACM Symposium on the Theory of Computing, pages 751-760. ACM, 2002.

[Sch83] U. Schürfeld. New lower bounds on the formula size of Boolean functions. Acta Informatica, 19(2):183-194, 1983.

[Sha49] C.E. Shannon. The synthesis of two-terminal switching circuits. Bell System Technical Journal, 28(1):59-98, 1949.

[Spi71] P. Spira. On time-hardware complexity tradeoffs for Boolean functions. In Proceedings of the 4th Hawaii Symposium on System Sciences, pages 525-527. Western Periodicals Company, North Hollywood, 1971. 
[ŠS04] R. Špalek and M. Szegedy. All quantum adversary methods are equivalent. Technical Report 0409116, quant-ph, 2004. quant-ph/0409116

[SV01] P. Sen and S. Venkatesh. Lower bounds in the quantum cell probe model. In Proceedings of Intenational Colloquium on Automata, Languages and Programming (ICALP), pages 358-369, 2001.

[Sze03] M. Szegedy. An $O\left(n^{1.3}\right)$ quantum algorithm for the triangle finding problem. Technical Report 0310134, quant-ph, 2003. quant-ph/0310134

[Val84] L.G. Valiant. Short monotone formulae for the majority function. Journal of Algorithms, 5:363-366, 1984.

[Weg87] I. Wegener. The Complexity of Boolean Functions. Wiley-Teubner, 1987.

[Zha04] S. Zhang. On the power of Ambainis's lower bounds. In Proceedings of 31st International Colloquium on Automata, Languages and Programming, 2004. To appear. Also in quant-ph/0311060

[Zwi91] U. Zwick. An extension of Khrapchenko's theorem. Information Processing Letters, 37(4):215-217, 1991. 